\title{
Chromatic coupling correction in the Large Hadron Collider
}

\author{
T. H. B. Persson, ${ }^{1,2}$ Y. Inntjore Levinsen, ${ }^{1}$ R. Tomás, ${ }^{1}$ and E. H. Maclean ${ }^{1,3}$ \\ ${ }^{1}$ CERN, CH-1211 Geneva 23, Switzerland \\ ${ }^{2}$ Chalmers University of Technology, 41296 Gothenburg, Sweden \\ ${ }^{3}$ John Adams Institute for Accelerator Science, Department of Particle Physics, University of Oxford, \\ Denys Wilkinson Building, Keble Road, Oxford, OX1 3RH, United Kingdom
}

(Received 13 June 2013; published 26 August 2013)

\begin{abstract}
In this article we present the measurement and correction of the chromatic coupling in the LHC. The transverse coupling is calculated from turn-by-turn data, exciting the beam with an ac dipole. By adjusting the momentum relative to the reference energy and repeating the measurement, we obtain the transverse coupling dependency on momentum. The measurements are compared to the predictions from the magnetic model, and show good agreement. We describe the algorithm used to calculate the corrections, which are performed using skew sextupoles in the LHC lattice. A reduction in the chromatic coupling of a factor $2.5 \pm 0.4$ for beam 1 and $1.6 \pm 0.3$ for beam 2 was obtained after applying the corrections.
\end{abstract}

DOI: 10.1103/PhysRevSTAB.16.081003

\section{INTRODUCTION}

The first beams were delivered to LHC in 2008, with first collisions the following year. The LHC design presents novel requirements for optics distortions. Extensive care has been taken to properly understand and correct the $\beta$-beat, betatron coupling, and other linear errors [1-3]. Controlling these errors is essential for a well-understood and predictable machine, and helps to reduce orbit distortions and improve beam quality and lifetime.

With the linear optics under control, the natural progression is to consider correction of the chromatic functions and other nonlinear contributions, which may result in significant improvement of beam and emittance lifetime. In the following we present the first measurements and corrections of the chromatic coupling in the LHC.

Chromatic coupling is generated by sextupolar errors in the magnets, in combination with dispersion. An offmomentum particle will experience a skew quadrupolar field from a sextupole in dispersive regions (vertical dispersion and normal sextupole, or horizontal dispersion and skew sextupole). Since there is larger horizontal dispersion in a ring, skew sextupolar components are the dominant source of chromatic coupling.

Improving chromatic coupling (and other higher order error components) will generally improve lifetime and control of the beam. Chromatic coupling creates a dependency between the tune and the momentum, which deteriorates the beam quality and reduces the available dynamic aperture [4,5]. An increase in luminosity of $20 \%$ after local chromatic coupling correction of the IP in KEKB was

Published by the American Physical Society under the terms of the Creative Commons Attribution 3.0 License. Further distribution of this work must maintain attribution to the author(s) and the published article's title, journal citation, and DOI.
PACS numbers: 41.85. $-\mathrm{p}, 41.75 .-\mathrm{i}, 29.20 . \mathrm{D}-$, 29.27.-a

reported in [6,7]. The effect of a correction is not expected to be as pronounced for LHC since round beams are collided instead of flat beams. In some of the future operation scenarios, however, flat beams are considered for the LHC [8]. To prepare for such a scenario it is important to demonstrate that we can measure and control the chromatic coupling.

Linear transverse coupling originates from skew quadrupolar errors and can be described by the resonance driving terms $f_{1001}$ (difference coupling) and $f_{1010}$ (sum coupling). The coupling terms are derived using Hamiltonian and normal form theory and the coupled lattice is treated as a perturbation to the uncoupled lattice. These driving terms relate to the normalized coupling matrix $\bar{C}$ by the relations [9]

$$
\begin{gathered}
f_{1001}=\frac{1}{4 \gamma}\left[\bar{C}_{12}-\bar{C}_{21}+i\left(\bar{C}_{11}+\bar{C}_{22}\right)\right], \\
f_{1010}=-\frac{1}{4 \gamma}\left[\bar{C}_{12}+\bar{C}_{21}-i\left(\bar{C}_{11}-\bar{C}_{22}\right)\right],
\end{gathered}
$$

where

$$
\gamma^{2}=1-|\bar{C}|
$$

The resonance condition for the difference coupling is that the tune difference in the two transverse planes should be close to an integer, while for the sum coupling the resonance condition is that the sum of the tune should be close to an integer. In this paper we look at how these driving terms vary with momentum change.

It was shown early on for the LHC studies that the chromatic coupling would be a significant source of perturbation at injection $[4,10,11]$, caused by a strong $a_{3}$ component in the arc dipoles. For this reason, skew sextupoles were installed in all arcs to compensate for chromatic coupling as shown in Fig. 1. The four skew sextupoles in one arc belong to one family (they share the same power 


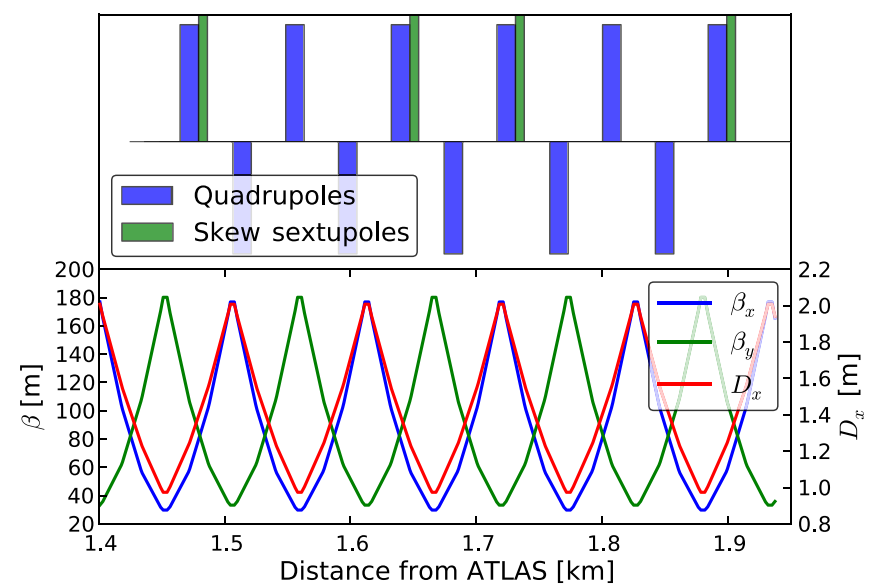

FIG. 1. Top: quadrupoles and skew sextupoles in the LHC arc. There are four skew sextupoles installed in each arc to correct for the chromatic coupling, shown as green bars. The horizontal axis shows the distance from ATLAS, so this is in the middle of the arc. Bottom: $\beta$ functions and horizontal dispersion. The phase advance between each FODO cell is approximately $90^{\circ}$.

supply). Between each $\beta$ peak (FODO cell) there is a phase advance of about $90^{\circ}$. Hence the phase advance between the four skew sextupoles is $180^{\circ}-90^{\circ}-180^{\circ}$. As a result, the locations (phase advances) of the skew sextupoles are good for correction of the chromatic difference coupling $\left(f_{1001}\right)$, while the chromatic sum coupling $\left(f_{1010}\right)$ will stay untouched. Since the four sextupoles are in the same family, the correction of the sum coupling performed by the first two sextupoles will for the most part be cancelled by the latter two.

\section{MEASURING CHROMATIC COUPLING}

The nominal fractional tunes in the LHC are $Q_{x}=0.31$ and $Q_{y}=0.32$ in collision, and the injection tunes are $Q_{x}=$ 0.28 and $Q_{y}=0.31$. As a result the difference coupling term is the dominant when compared to the sum coupling $\left(f_{1001} \gg f_{1010}\right)$. In this article we will therefore focus on measurement and correction of the chromatic $f_{1001}$, but the same approach is valid for the chromatic sum resonance. In this paper we define the chromatic coupling as

$$
\left|C_{c h}^{-}\right| \equiv\left|\frac{\partial C^{-}}{\partial \delta}\right| \approx 4 \Delta Q\left|\frac{\Delta \bar{f}_{1001}}{\Delta \delta}\right|,
$$

where $\delta$ is the momentum, and $\Delta Q$ is the difference of the fractional tunes between the two planes. The relation between $C^{-}$and the resonance driving term $f_{1001}$ was shown for linear coupling in [12]. We are considering a linear dependency between the coupling and momentum, which is expected to be the dominant part of the chromatic dependency on the coupling. The beam is excited using an ac dipole and $\sim 2000$ turns of beam position monitor (BPM) data are recorded while the dipole is at flat top. More details about the measurement technique can be found in $[13,14]$. The turn-by-turn data from two consecutive BPMs are used to
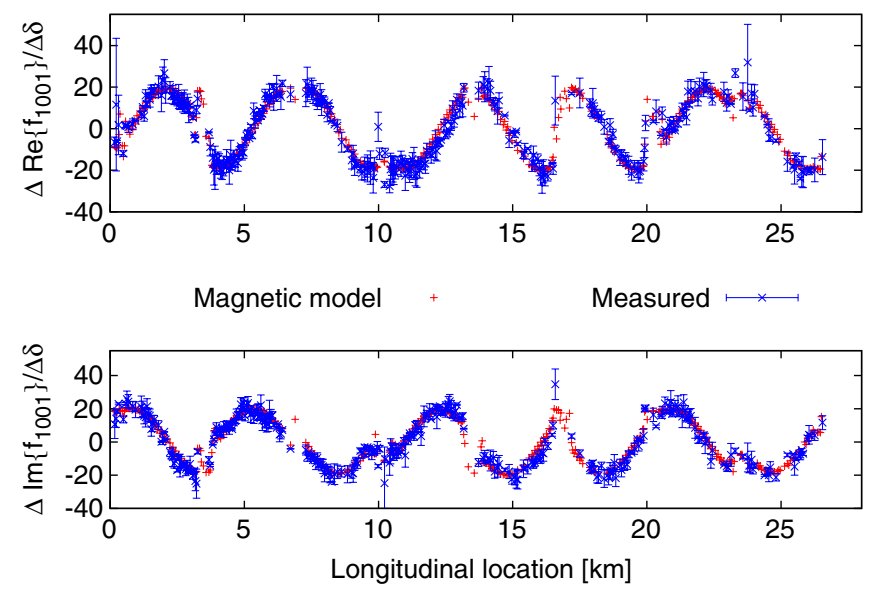

FIG. 2. Comparison between the measured chromatic coupling (blue) and the expected chromatic coupling from our magnetic model (red). This is for beam 1 at injection.

calculate the $f_{1001}$ [15]. We have usually conducted a correction of the on-momentum coupling prior to measurements of the chromatic coupling. It has been found in both measurements and simulations that an on-momentum coupling will add a beating to our chromatic coupling signal (see Fig. 5 later), and if very strong, deteriorate the measurement significantly.

We measure the coupling for on-momentum and with $\pm \delta p$, where $\delta p$ is typically around $5-10 \times 10^{-4}$. This gives the coupling for three different momenta at each BPM, and we make a linear regression fit to get the chromatic coupling.

In Fig. 2, we see an example of the comparison at injection optics between the measured chromatic coupling and our expectations based on the available knowledge of the magnetic field errors [16]. The measurement is in excellent agreement with our expectations, demonstrating that the dominant contributions to chromatic coupling are known.
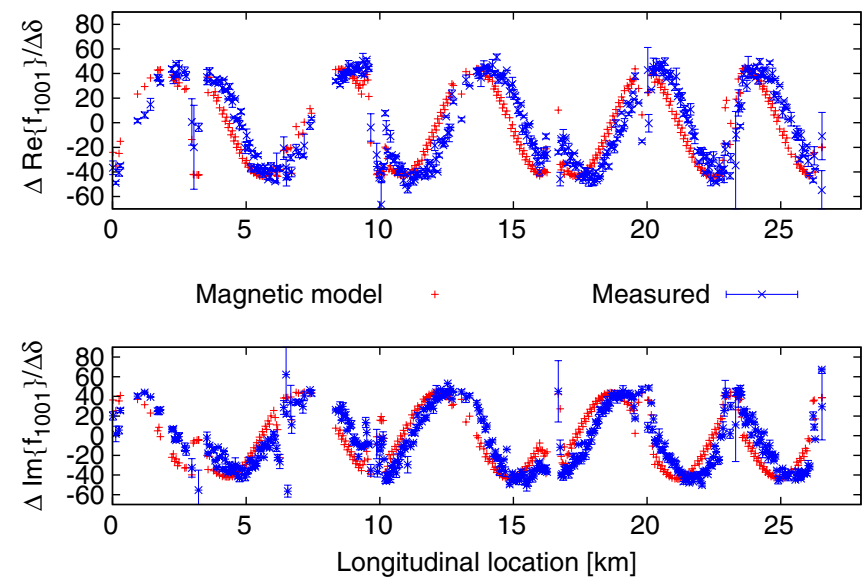

FIG. 3. Comparison between the measured chromatic coupling (blue) and the expected chromatic coupling from our magnetic model (red). This is for beam 2 at $4 \mathrm{TeV}$, with a $\beta^{*}$ of $60 \mathrm{~cm}$. 

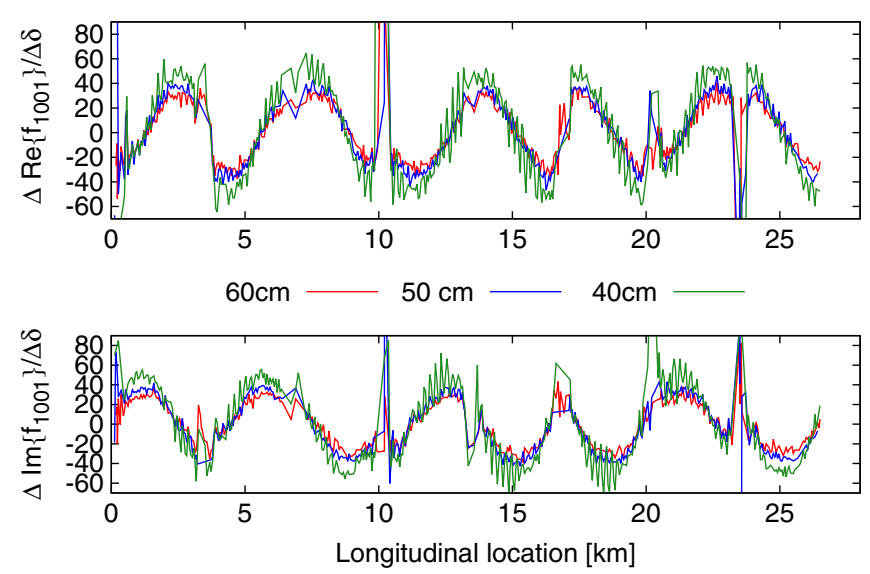

FIG. 4. The measured beam 1 chromatic coupling for different $\beta^{*}$ at $4 \mathrm{TeV}$.

In Fig. 3 a similar comparison for squeezed optics and $4 \mathrm{TeV}$ beam energy is shown. We see once more a very good agreement. Only a small phase shift is noticeable. A phase shift indicates that the errors are distributed slightly differently compared to the model or that there is a small deviation of the betatron phase advance. The phase shift is not visible for the injection optics which indicates that the small discrepancy is related to the $\beta^{*}$. When the beam is squeezed the $\beta$ function inside the triplet is increased to $4 \mathrm{~km}$ which is $\sim 20$ times larger than at injection. As a consequence any error in the triplet will be more evident for the $\beta^{*}$ of $60 \mathrm{~cm}$ compared to injection optics. This is a possible explanation as to why the small phase shift is only visible for the squeezed optics.

Note that although the $f_{1001}$ is changing more per momentum deviation for squeezed optics, the $C_{c h}^{-}$is actually smaller due to the smaller tune split at $\beta^{*}$ of $60 \mathrm{~cm}$; see Eq. (4).

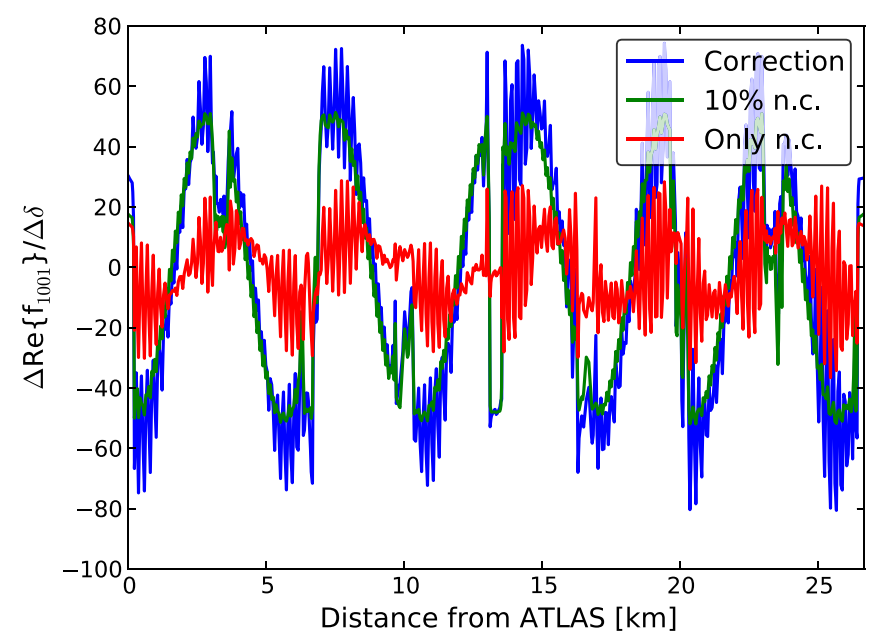

FIG. 5. The chromatic coupling from our model, with corrections for normal (skew quadrupoles) and chromatic coupling (skew sextupoles) added. The green line shows the same correctors added, but only $10 \%$ of the strength in the skew quadrupoles. The red line shows the chromatic coupling coming only from the skew quadrupoles.
In Fig. 4 we see the chromatic coupling for different $\beta^{*}$. A slight increase is observed, but 60 and $50 \mathrm{~cm}$ are within the error bars. At $40 \mathrm{~cm}$ we see a significant increase. A beating is also added to the signal for $40 \mathrm{~cm}$, which based on our model predictions points towards a residual on-momentum coupling left in the machine. This is shown in Fig. 5. The green line in this figure shows that the beating disappears when we take away the on-momentum coupling (skew quadrupoles).

The large spikes shown in Fig. 4 at around 10 and $23 \mathrm{~km}$ are CMS and ATLAS interaction points, respectively. Here the measurement uncertainty is large (not shown), due to a small phase advance between the BPMs at these locations.

\section{CORRECTION ALGORITHM}

The correction algorithm is based on a matrix inversion approach. A response matrix $\mathbf{R}$ is created using the ideal model. The matrix relates the chromatic coupling at $n$ BPMs to the strength of the available skew sextupole circuits $m$. This results in an $m \times n$ response matrix:

$$
\mathbf{R} \Delta K_{s s}=\left(\operatorname{Re}\left\{\frac{\Delta \vec{f}_{1001}}{\Delta \delta}\right\}, \operatorname{Im}\left\{\frac{\Delta \vec{f}_{1001}}{\Delta \delta}\right\}\right) .
$$

The measured chromatic coupling is then multiplied with the generalized inverted matrix, $\mathbf{R}^{-1}$, to calculate the correction strength. The system is overconstrained but it is possible to solve this with a least-square scheme using singular value decomposition,

$$
\Delta K_{s s}=\mathbf{R}^{-1}\left(\operatorname{Re}\left\{\frac{\Delta \vec{f}_{1001}}{\Delta \delta}\right\}, \operatorname{Im}\left\{\frac{\Delta \vec{f}_{1001}}{\Delta \delta}\right\}\right)
$$

Locations with large measurement errors are filtered out before the correction is calculated.

A quality control of the correction is performed before its application in the machine. The predicted correction is simulated using the ideal model with the calculated
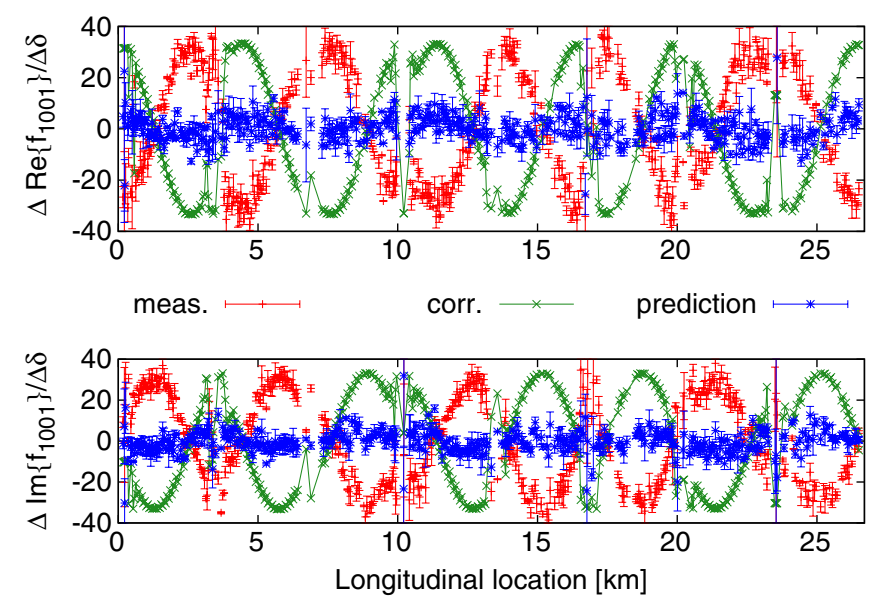

FIG. 6. The measurement (red), correction (green), and predicted chromatic coupling after correction (blue), for beam 1 at $4 \mathrm{TeV}$ and $\mathrm{a} \beta^{*}$ of $60 \mathrm{~cm}$. 
TABLE I. The calculated knob values from the two algorithms. Note that for the response matrix algorithm, we excluded the corrector in the last arc.

\begin{tabular}{lcccc}
\hline \hline & \multicolumn{2}{c}{ Response matrix } & \multicolumn{2}{c}{ Arc-by-arc } \\
& Beam 1 & Beam 2 & Beam 1 & Beam 2 \\
Arc & & {$\left[10^{-3} \mathrm{~m}^{-3}\right]$} & \\
\hline 12 & -7.57 & -10.5 & -52.3 & 54.5 \\
23 & 8.8 & 10.2 & -33.6 & 36.6 \\
34 & 2.79 & 0.296 & -32.6 & 31.3 \\
45 & -4.9 & -6.95 & -31.3 & 24 \\
56 & -0.319 & 2.39 & -2.15 & 3.86 \\
67 & -7.76 & -9.85 & -6.84 & 2.26 \\
78 & -5.77 & -7 & -35.6 & 33.6 \\
81 & - & 9.89 & -15.3 & 18.3 \\
\hline \hline
\end{tabular}

strength of the skew sextupoles. The calculated correction for beam 1 is shown in Fig. 6 together with the measured data. The expected residual chromatic coupling after correction is also shown.

An existing "arc-by-arc" correction algorithm was implemented earlier [17], and a correction has been calculated from this algorithm based on the magnetic model data. The corrections calculated from both algorithms are similar in their effect on the chromatic coupling. The powering of the skew sextupoles, however, are very different, as can be seen in Table I. The arc-by-arc algorithm first applies a local correction in each arc, before doing a global correction of the residual chromatic coupling. This means that the cancellation between the arcs is not exploited as well as with a purely global correction. As a result the rms strength of the skew sextupoles from the arc-by-arc method is approximately 5 times higher for beam 1 and 4 times higher for beam 2 compared to the response matrix algorithm. The main objective of the correction is to reduce the $C_{c h}^{-}$. The corrections based on the response matrix are preferred, since they require less strength of the skew sextupoles.

\section{RESULTS OF CORRECTION}

In 2012 the first chromatic coupling correction was performed in the LHC [18]. The correction was tested for the nominal 2012 optics $\left(\beta^{*}=0.6 \mathrm{~m}\right)$. Beam 2 had eight independent skew sextupole circuits while beam 1 had seven available (one was out of commission). In Fig. 7 the chromatic coupling before and after correction is presented. The weighted mean value of the chromatic $f_{1001}$ before and after correction is shown in Table II. The chromatic $f_{1001}$ was decreased by $\sim 20$ units for both beams, proving the corrections successful.

The measured chromatic coupling after correction is compared to the predicted correction in Fig. 8. The predicted correction is based on the model and the measurement before the correction. The difference between the predicted and the measured corrections is for the most part within the error bars. The slight increase in measured

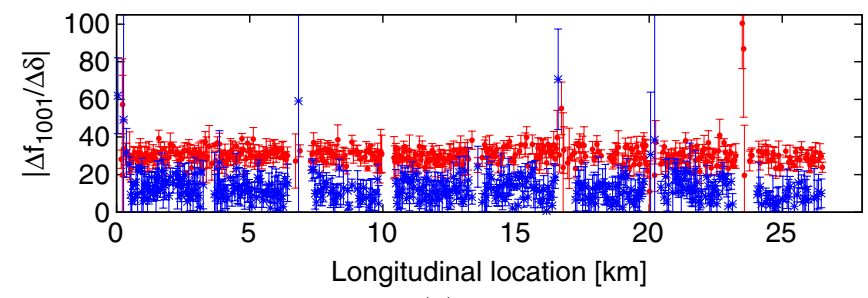

(a)Beam 1

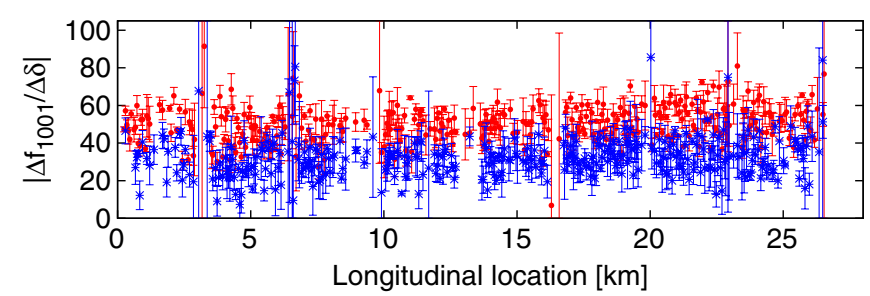

(b)Beam 2

FIG. 7. The absolute value of the chromatic coupling before (red) and after (blue) correction.
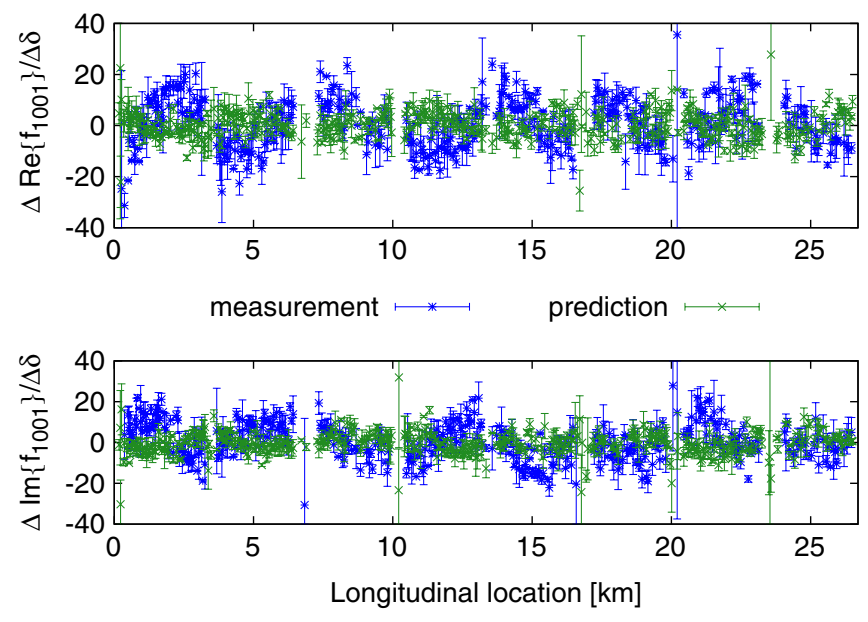

FIG. 8. Comparison between the measured chromatic coupling after correction (red) and the predicted chromatic coupling after correction from our model (green). This is for beam 1 at $\beta^{*}=0.6 \mathrm{~m}$.

chromatic coupling can be due to imperfections in the skew sextupoles used for the correction or a small horizontal dispersion discrepancy.

In order to reduce the chromatic coupling further, it is possible to calculate a second correction, based on the measurement after the first correction.

TABLE II. The weighted mean value of the chromatic $f_{1001}$ and its standard deviation, before and after correction, for beam 1 and beam 2 , respectively.

\begin{tabular}{lcccc}
\hline \hline & \multicolumn{2}{c}{ Before correction } & \multicolumn{2}{c}{ After correction } \\
& $\left|\frac{\Delta \bar{f}_{1001}}{\Delta \delta}\right|$ & Error $(\sigma)$ & $\left|\frac{\Delta \bar{f}_{1001} \mid}{\Delta \delta}\right|$ & Error $(\sigma)$ \\
\hline Beam 1 & 31.5 & 5.4 & 12.5 & 5.3 \\
Beam 2 & 50.1 & 8.5 & 31.4 & 9.0 \\
\hline \hline
\end{tabular}




\section{CONCLUSION}

A successful correction of the chromatic coupling has been demonstrated for the first time in the LHC. The corrections implemented are based on the use of skew sextupoles. Their optimum settings have been calculated from beam based measurements.

The measured chromatic coupling has been shown to be in good agreement with predictions from the magnetic model. The strength of the correction obtained from the beam based measurement is more than a factor 4 lower compared to the correction calculated from the previous arc-by-arc algorithm. The corrections were not used so far during normal operation, so the impact on the overall machine performance remains to be seen.

\section{ACKNOWLEDGMENTS}

The authors would like to thank T. Bach, A. Langner, M. McAteer, P. K. Skowronski, and S. M. White for a very fruitful collaboration during measurements. Further, a big thanks to the LHC operation for excellent conditions to carry out our measurements. Finally we would like to acknowledge the fruitful discussions and input from $\mathrm{M}$. Giovannozzi and S. Fartoukh.

[1] M. Aiba et al., Phys. Rev. ST Accel. Beams 12, 081002 (2009).

[2] R. Tomás, O. Brüning, M. Giovannozzi, P. Hagen, M. Lamont, F. Schmidt, G. Vanbavinckhove, M. Aiba, R. Calaga, and R. Miyamoto, Phys. Rev. ST Accel. Beams 13, 121004 (2010).

[3] R. Tomás, T. Bach, R. Calaga, A. Langner, Y. I. Levinsen, E. H. Maclean, T.H. B. Persson, P. K. Skowronski, M. Strzelczyk, G. Vanbavinckhove, and R. Miyamoto, Phys. Rev. ST Accel. Beams 15, 091001 (2012).

[4] S. D. Fartoukh, CERN, Geneva Technical Report No. CERN-LHC-Project-Report-278, 1999 [http://cdsweb .cern.ch/record/383926].
[5] S.D. Fartoukh and M. Giovannozzi, Nucl. Instrum. Methods Phys. Res., Sect. A 671, 10 (2012).

[6] Y. Ohnishi, K. Ohmi, H. Koiso, M. Masuzawa, A. Morita, K. Mori, K. Oide, Y. Seimiya, and D. Zhou, Phys. Rev. ST Accel. Beams 12, 091002 (2009).

[7] D. Zhou, K. Ohmi, Y. Seimiya, Y. Ohnishi, and A. Morita, Technical Report No. 2009-10, 2009 [http://wwwlib.kek.jp/cgi-bin/kiss_prepri.v8?KN=200927010\&OF=8].

[8] S. Fartoukh and R. De Maria, in Proceedings of the 3rd International Particle Accelerator Conference, New Orleans, Louisiana, USA, 2012 (IEEE, Piscataway, NJ, 2012), Report No. CERN-ATS-2012-136 [http:// cds.cern.ch/record/1459916].

[9] R. Calaga, R. Tomás, and A. Franchi, Phys. Rev. ST Accel. Beams 8, 034001 (2005).

[10] J.-P. Koutchouk, CERN, Geneva Technical Report No. CERN-LHC-Project-Note-113, 1997 [http://cdsweb .cern.ch/record/691854].

[11] S. D. Fartoukh and J.-P. Koutchouk, in Proceedings of the European Particle Accelerator Conference, Vienna, 2000 (EPS, Geneva, 2000), Report No. CERN-LHC-ProjectReport-400, p. 4 [http://cdsweb.cern.ch/record/461473].

[12] A. Franchi, Ph.D. thesis, GSI DISS, Johann Wolfgang Goethe-Universität Frankfurt am Main, 2006 [http:// www-alt.gsi.de/documents/DOC-2007-Jun-38-1.pdf].

[13] R. Miyamoto, M. Aiba, R. Calaga, R. Tomás, and G. Vanbavinckhove, in Proceedings of the 2nd International Particle Accelerator Conference, San Sebastián, Spain (EPS-AG, Spain, 2011), WEPC030 [http://accelconf.web.cern.ch/accelconf/IPAC2011/papers/ wepc030.pdf].

[14] R. Miyamoto, Technical Report No. C-A/AP\#410, 2010 [http://www.bnl.gov/isd/documents/74582.pdf].

[15] A. Franchi, R. Tomás, and G. Vanbavinkhove, Technical Report No. CERN-BE-Note-2010-016, 2010 [https:// cds.cern.ch/record/1264111].

[16] N. J. Sammut, L. Bottura, and J. Micallef, CERN Report No. CERN-LHC-Project-Report-839 (2005), p. 4 [http:// cds.cern.ch/record/865945].

[17] S. D. Fartoukh (private communication).

[18] T. Bach et al., CERN, Geneva Technical Report No. CERN-ACC-NOTE-2013-0004 MD, 2013 [https:// cds.cern.ch/record/1571167]. 